\title{
Erratum to: Favourable effects of fenofibrate on lipids and cardiovascular disease in women with type 2 diabetes: results from the Fenofibrate Intervention and Event Lowering in Diabetes (FIELD) study
}

\author{
Michael C. d'Emden ${ }^{1,2}$ • Alicia J. Jenkins ${ }^{4} \cdot$ Liping $\mathrm{Li}^{3} \cdot$ Diana Zannino ${ }^{3}$ • \\ Kristy P. Mann ${ }^{3}$ - James D. Best ${ }^{4}$ - Bronwyn G. A. Stuckey ${ }^{5}$ Kris Park ${ }^{6}$. \\ Juha Saltevo $^{7}$ • Anthony C. Keech ${ }^{3} \cdot$ on behalf of the FIELD Study Investigators
}

Published online: 18 July 2015

(C) Springer-Verlag Berlin Heidelberg 2015

\section{Erratum to: Diabetologia}

DOI 10.1007/s00125-014-3344-3

The authors wish to point out two typographical errors in Fig. 3. In the fenofibrate arm, the proportion of men who had a stroke was $3.8 \%$ (not $13.8 \%$ ) and the proportion of men who underwent revascularisation was $9.9 \%$ (not $39.9 \%$ ). The plotted hazard ratios are correct.

The online version of the original article can be found at http://dx.doi.org/ 10.1007/s00125-014-3344-3.

Anthony C. Keech

fieldtrial@ctc.usyd.edu.au

1 Endocrine Research Unit, Royal Brisbane Hospital, Brisbane, QLD, Australia

2 Department of Medicine, University of Queensland, Brisbane, QLD, Australia

3 National Health and Medical Research Council Clinical Trials Centre, University of Sydney, Locked Bag 77, Camperdown, Sydney, NSW 1450, Australia

4 Department of Medicine, University of Melbourne, Melbourne, VIC, Australia

5 Keogh Institute for Medical Research and Sir Charles Gairdner Hospital, Perth, WA, Australia

6 Nepean Hospital, Sydney, NSW, Australia

7 Department of Medicine, Central Finland Central Hospital, Jyväskylä, Finland 